\title{
Lie symmetry analysis for the solution of first-order linear and nonlinear fractional differential equations
}

\author{
Mousa Ilie 1, 2, Jafar Biazar 2,3*, Zainab Ayati ${ }^{4}$ \\ ${ }^{1}$ Department of Mathematics, Guilan Science and Research Branch, Islamic Azad University, Rasht, Iran \\ ${ }^{2}$ Department of Mathematics, Rasht Branch, Islamic Azad University, Rasht, Iran \\ ${ }^{3}$ Department of Applied Mathematics, Faculty of Mathematical Sciences, University of Guilan, \\ P.O. Box. 41335-1914, Guilan, Rasht, Iran \\ ${ }^{4}$ Department of Engineering sciences, Faculty of Technology and Engineering East of Guilan, \\ University of Guilan, P.C. 44891-63157, Rudsar-Vajargah, Iran \\ *Corresponding author E-mail: biazar@iaurasht.ac.ir
}

\begin{abstract}
Obtaining analytical or numerical solution of fractional differential equations is one of the troublesome and challenging issues among mathematicians and engineers, specifically in recent years. The purpose of this paper is to solve linear and nonlinear fractional differential equations such as first order linear fractional equation, Bernoulli, and Riccati fractional equations by using Lie Symmetry method, based on conformable fractional derivative. For each equation, some numerical examples are presented to illustrate the proposed approach.
\end{abstract}

Keywords: Linear and Nonlinear Fractional Equations; Lie Symmetry Method; Conformable Fractional Derivative; Bernoulli Fractional Equation; Riccati Fractional Equation.

\section{Introduction}

Fractional calculus is as old as the usual calculus. In the past several years, many of researchers have been trying to generalize the concept of the usual derivatives. Nowadays there are many definitions for the fractional derivative. Two the earliest of definitions are as follows (see [1]):

i) Riemann-Liouville definition: If $\mathrm{n}$ is a positive integer and $\alpha \in[n-1, n)$ the $\alpha$-th derivative of $f$ is given by

$D_{a}^{\alpha}(f)(x)=\frac{1}{\Gamma(n-\alpha)} \frac{d^{n}}{d x^{n}} \int_{a}^{x} \frac{f(t)}{(x-t)^{\alpha-n+1}} d t$.

ii) Caputo definition: For $\alpha \in[\mathrm{n}-1, \mathrm{n})$ the $\alpha$-th derivative of $\mathrm{f}$ is

$D_{a}^{\alpha}(f)(x)=\frac{1}{\Gamma(n-\alpha)} \int_{a}^{x} \frac{f^{n}(t)}{(x-t)^{\alpha-n+1}} d t$

The presented definitions are attempted to satisfy the usual properties of the standard derivative (see [1]). The only property inherited by all definitions of fractional derivative is the linearity property. But there are some disadvantages that caused their application confront with difficulty (see [1]).

One of the definition that have been presented recently is conformable fractional derivative that removed some of drawbacks the presented definitions.

Consider a function $f:[0, \infty) \rightarrow \mathbb{R}$. Then conformable fractional derivative of $f$ of order $\alpha$ is defined by
$T_{\alpha}(f)(x)=\lim _{\varepsilon \rightarrow 0} \frac{f\left(x+\varepsilon x^{1-\alpha}\right)-f(x)}{\varepsilon}$

for all $x>0, \alpha \in(0,1]$. If $\mathrm{f}$ is $\alpha$ - differentiable in some $(0, a), a>$ 0 , and $\lim _{x \rightarrow 0^{+}} T_{\alpha}(f)(x)$ exists, then one can define $T_{\alpha}(f)(0)=$ $\lim _{x \rightarrow 0^{+}} T_{\alpha}(f)(x)$.

If the conformable derivative of $\mathrm{f}$ of order $\alpha$ exists, then we simply say that $f$ is $\alpha$-differentiable (see [1], [2]).

One can easily show that $T_{\alpha}$ satisfies all the following properties:

Let $\alpha \in(0,1]$ and be $\alpha$-differentiable at a point $x>0$, Then (see [1])

1) For $a, b \in \mathbb{R} T_{\alpha}(a f+b g)=a T_{\alpha}(f)+b T_{\alpha}(g)$,

2) For all $p \in \mathbb{R} T_{\alpha}\left(x^{p}\right)=p x^{p-\alpha}$,

3) For all constant functions $f(x)=\lambda, T_{\alpha}(\lambda)=0$,

4) $T_{\alpha}(f \cdot g)=g \cdot T_{\alpha}(f)+f \cdot T_{\alpha}(g)$,

5) $T_{\alpha}\left(\frac{f}{g}\right)=\frac{g \cdot T_{\alpha}(f)-f \cdot T_{\alpha}(g)}{g^{2}}$,

6) $T_{\alpha}(f)=x^{1-\alpha} \frac{d f}{d x}$.

Whereas solving fractional differential equations is very important, there are many fractional differential equations which can't be solved analytically. Due to this fact, finding an approximate solution of fractional differential equations is clearly an important task. In recent years, many effective methods have been proposed for finding approximate solution to fractional differential equations [620]. The purpose of this paper is solving fractional equation by Lie Symmetry method, based on conformable fractional derivative. 
The organization of this paper is as follows: In Section 2, Lie invariance condition will be described. In subsection 3.1, 3.2, and 3.3, respectively the method will be used to solve first order linear fractional equation, Bernoulli fractional equation, and Riccati fractional equation. For each equation there are some examples, as well. Finally, discussion will be given, in section 4 .

\section{Lie symmetry method}

Let consider the invariance of

$\frac{d y}{d x}=F(x, y)$,

Under the infinitesimal transformation

$\bar{x}=x+X(x, y) \varepsilon+O\left(\varepsilon^{2}\right)$,

$\bar{y}=y+Y(x, y) \varepsilon+O\left(\varepsilon^{2}\right)$.

The derivatives transform under the infinitesimal transformations (2.2) is as follows (see [3-5])

$\frac{d \bar{y}}{d \bar{x}}=\frac{d y}{d x}+\left(\frac{\partial Y}{\partial x}+\left[\frac{\partial Y}{\partial y}-\frac{\partial X}{\partial x}\right] \frac{d y}{d x}-\frac{\partial X}{\partial y}\left(\frac{d y}{d x}\right)^{2}\right) \varepsilon+O\left(\varepsilon^{2}\right)$.

Consider the following ODE

$\frac{d \bar{y}}{d \bar{x}}=F(\bar{x}, \bar{y})$.

Substituting the infinitesimal transformations (2.2) and first-order derivative transformation (2.3) into (2.4) yields

$\frac{d y}{d x}+\left(\frac{\partial Y}{\partial x}+\left[\frac{\partial Y}{\partial y}-\frac{\partial X}{\partial x}\right] \frac{d y}{d x}-\frac{\partial X}{\partial y}\left(\frac{d y}{d x}\right)^{2}\right) \varepsilon+O\left(\varepsilon^{2}\right)=F(x+$ $\left.X(x, y) \varepsilon+O\left(\varepsilon^{2}\right), y+Y(x, y) \varepsilon+O\left(\varepsilon^{2}\right)\right)$.

Expanding to order $O\left(\varepsilon^{2}\right)$ gives

$\frac{d y}{d x}+\left(\frac{\partial Y}{\partial x}+\left[\frac{\partial Y}{\partial y}-\frac{\partial X}{\partial x}\right] \frac{d y}{d x}-\frac{\partial X}{\partial y}\left(\frac{d y}{d x}\right)^{2}\right) \varepsilon+O\left(\varepsilon^{2}\right)=$

$F(x, y)+\left(X \frac{\partial F}{\partial x}+Y \frac{\partial F}{\partial y}\right) \varepsilon+O\left(\varepsilon^{2}\right)$,

By using (2.1), Eq. (2.5) is satisfied to $O\left(\varepsilon^{2}\right)$ if

$\frac{\partial Y}{\partial x}+\left[\frac{\partial Y}{\partial y}-\frac{\partial X}{\partial x}\right] F-\frac{\partial X}{\partial y} F^{2}=X \frac{\partial F}{\partial x}+Y \frac{\partial F}{\partial y}$

This is known as Lie's Invariance Condition. For a given $F(x, y)$, any functions $X(x, y)$ and $Y(x, y)$ that solve equation (2.6) are the infinitesimals (see [3-5]).

A transformation can be constructed that would lead to a separable equation involving $r$, and $s$. Consider

$r=r(x, y), s=s(x, y)$

and require that (2.7) be invariant, that is,

$\bar{r}=r(\bar{x}, \bar{y}), \bar{s}=s(\bar{x}, \bar{y})$

The separable equation

$\frac{d s}{d r}=G(r)$

Is invariant under

$\bar{r}=r, \bar{s}=s+\varepsilon$.

Differentiating (2.8) with respect to $\varepsilon$ and setting $\varepsilon=0$, and using Eqs. (2.9), gives
$X(x, y) \frac{\partial r}{\partial x}+Y(x, y) \frac{\partial r}{\partial y}=0$,

$X(x, y) \frac{\partial s}{\partial x}+Y(x, y) \frac{\partial s}{\partial y}=1$.

Thus, if the infinitesimals $X$ and $Y$ are had, solving (2.10) would give rise to the transformation that will separate the given ordinary differential equation (2.1) (see [3-5]).

\section{Applications of lie symmetry method}

In this section by using lie symmetry method, presented a general solution for linear and nonlinear first order fractional differential equations.

\subsection{Solving first order linear fractional differential equa- tions}

General form of a first order linear fractional equations is as follow,

$T_{\alpha}(y)(x)+p(x) y(x)=q(x)$

Where $p(x), q(x)$ are $\alpha$-differentiable functions, and $y(x)$ is an unknown function. By using the property (6), Eq. (3.1) can be written as the following form

$x^{1-\alpha} y^{\prime}(x)+p(x) y(x)=q(x)$

So

$y^{\prime}(x)+P(x) y(x)=Q(x)$

Where $P(x)=x^{\alpha-1} p(x)$ and $Q(x)=x^{\alpha-1} q(x)$. Eq. (3.2) is a first order linear ordinary differential equation. This is invariant under the Lie group

$\bar{x}=x, \bar{y}=y+\varepsilon e^{-\int P(x) d x}$,

Gives

$\frac{d y}{d x}-\varepsilon P(x) e^{-\int P(x) d x}+P(x)\left(y+\varepsilon e^{-\int P(x) d x}\right)=Q(x)$.

Expanding (3.4) gives

$\frac{d y}{d x}+P(x) y(x)=Q(x)$.

From the lie group (3.3), we obtain the infinitesimals $X=0$ and $Y=e^{-\int P(x) d x}$. The change of variables are obtained by solving (2.10), as follows

$e^{-\int P(x) d x} \frac{\partial r}{\partial y}=0, e^{-\int P(x) d x} \frac{\partial s}{\partial y}=1$.

Thus

$r=R(x), s=y e^{\int P(x) d x}+S(x)$

Where $R(x)$ and $S(x)$ are arbitrary functions. Choosing $R(x)=x$ and $S(x)=0$ results in

$x=r, y=s e^{-\int P(r) d r}$,

By calculating $\frac{d y}{d x}$, we obtain

$\frac{d y}{d x}=\frac{d s}{d r} e^{-\int P(r) d r}-s P(r) e^{-\int P(r) d r}$.

And substituting into (3.2) and simplifying, the following result will be given. 
$\frac{d s}{d r}=Q(r) e^{\int P(r) d r}$.

Which (3.6) is a separable equation (see [3-5]).

Example 3. 1. Consider the following equation

$T_{\frac{2}{3}} y+\sqrt[3]{x} y=x \sqrt[3]{x}$

By using property (6), Eq. (3.7), can be rewritten as follows

$y^{\prime}+y=x$

That $P(x)=1, Q(x)=x$, thereupon by Lie Symmetry method

$x=r, y=s e^{-r}$.

$\frac{d s}{d r}=r e^{r}$

Which is the separable equation having a general solution is as follows

$s=r e^{r}-e^{r}+C$

$y=C e^{-x}+x-1$

Example 3.2. Consider the following equation

$T_{\frac{1}{2}} y-2 \sqrt{x} y=x \sqrt{x}$

By using property (6), we derive

$y^{\prime}-2 y=x$

From (3.5) and (3.6) the general solution of equation (3.11) is as the following

$y=C e^{2 x}-0.5 x-0.25$

\subsection{Solving bernoulli fractional equation}

The Bernoulli fractional differential equations have the following general form,

$T_{\alpha} y+p(x) y=q(x) y^{n}, n \neq 0,1$

Where $\alpha$-differentiable functions, $y(x)$ is an unknown function. By using property (6) Eq. (3.12) leads to

$x^{1-\alpha} y^{\prime}+p(x) y=q(x) y^{n}$

So,

$y^{\prime}(x)+P(x) y=Q(x) y^{n}$

Where $P(x)=x^{\alpha-1} p(x)$ and $Q(x)=x^{\alpha-1} q(x)$, equation (3.13) is the Bernoulli equation.

Assuming $X=0$, Lie's invariance condition becomes,

$\frac{\partial Y}{\partial x}+\frac{\partial Y}{\partial y}\left(Q(x) y^{n}-P(x) y\right)=Y\left(n Q(x) y^{n-1}-P(x)\right)$

That

$Y=e^{(n-1) \int P(x) d x} y^{n}$

Satisfies (3.14). To obtain a change of variables, it is necessary to solve $e^{(n-1) \int P(x) d x} y^{n} \frac{\partial r}{\partial y}=0, e^{(n-1) \int P(x) d x} y^{n} \frac{\partial s}{\partial y}=1$.

Example 3.3. Consider Bernoulli fractional equation

$T_{\frac{1}{2}} y+\frac{1}{\sqrt{x}} y=\sqrt{x} x y^{3}$

By using property (6), in equation (3.15), leads to

$y^{\prime}+\frac{1}{x} y=x y^{3}$

Here $P(x)=\frac{1}{x}, n=3$, choosing $R(x)=x$ and $S(x)=0$ which gives

$r=x, s=\frac{-1}{2 x^{2} y^{2}}$

Under this change of variables, the Bernoulli equation (3.17) turns to

$\frac{d s}{d r}=\frac{1}{r}$

Which is the separable equation and a general solution to this equation is as the following form

$s=\ln r+C$.

So the general solution of fractional equation (3.16) can be presented as follows

$2 x^{2} y^{2}(\ln x+C)-1=0$.

Example 3.4. Consider the following equation

$T_{\frac{2}{3}} y=2 \sqrt[3]{x} y+x \sqrt[3]{x} y^{2}$

Similar to previous example, we have

$y^{\prime}-2 y=x y^{2}$

Choosing $R(x)=x$, and $S(x)=0$, leads to

$r=x, s=\frac{-1}{y e^{-2 x}}$.

Under this change of variables, the Bernoulli equation (3.19) becomes

$\frac{d s}{d r}=r e^{2 r}$

The general solution of fractional equation (3.18) is as the following form

$y=\frac{-1}{C e^{-2 x}+0.5 x-0.25}$.

\subsection{Solving riccati fractional equation}

The general form of a Riccati fractional equation is as the following

$T_{\alpha} y=p(x) y^{2}+q(x) y+r(x)$

Where $\alpha$-differentiable functions and $y(x)$ is an unknown function. By using property (6), we obtain

$x^{1-\alpha} y^{\prime}=p(x) y^{2}+q(x) y+r(x)$,

So, 
$y^{\prime}=P(x) y^{2}+Q(x) y+R(x)$,

Where $P(x)=x^{\alpha-1} p(x), Q(x)=x^{\alpha-1} q(x) \quad$ and $\quad R(x)=$ $x^{\alpha-1} r(x)$. Equation (3.22) is the Riccati equation. We will assume $X=0$, giving Lie's invariance condition as

$\frac{\partial Y}{\partial x}+\left(P(x) y^{2}+Q(x) y+R(x)\right) \frac{\partial Y}{\partial y}=(2 P(x) y+Q(x)) Y$.

One solution of (3.23) is

$Y=\left(y-y_{1}\right)^{2} F(x)$

Where $y_{1}$ is one solution to (3.22) and F satisfies

$F^{\prime}+\left(2 P y_{1}+Q\right) F=0$.

Variables $r$ and $s$, it is necessary to solve

$\left(y-y_{1}\right)^{2} F(x) \frac{\partial r}{\partial y}=0,\left(y-y_{1}\right)^{2} F(x) \frac{\partial s}{\partial y}=1$,

From which we obtain

$r=K(x), s=S(x)-\frac{1}{\left(y-y_{1}\right) F}$,

Where $K(x)$ and $S(x)$ are arbitrary functions. Setting $K(x)=x$ and $S(x)=0$, yields to

$x=r, y=y_{1}-\frac{1}{s F(r)}$,

Thereby transforming the original Riccati equation (3.22) to

$\frac{d s}{d r}=\frac{a(r)}{F(r)}$.

It is interesting that the usual linearizing transformation is recovered using Lie Symmetry method (see [3-5]).

Example 3.5. Consider Riccati fractional equation as follows

$T_{\frac{1}{2}} y=\sqrt{x} y^{2}-\frac{1}{\sqrt{x}} y-\frac{1}{x \sqrt{x}}$,

That has a solution such as $y_{1}=-x^{-1}$.

Clearly by using property (6), this equation changing to

$y^{\prime}=y^{2}-\frac{1}{x} y-\frac{1}{x^{2}}$.

From (3.24) $\mathrm{F}$ is as the following form

$F(x)=x^{3}$,

Thus, under the change of variables given in (3.25), namely

$x=r, y=-\frac{1}{r}-\frac{1}{s r^{3}}$,

The original ODE becomes

$\frac{d s}{d r}=\frac{1}{r^{3}}$.

Which is a separable equation having a general solution is as follows

$s=-\frac{1}{2 r^{2}}+C$,

And the general solution of equation (3.26), can be presented as the following form

$y=-\frac{1}{x}-\frac{1}{C x^{3}-0.5 x}$
Example 3.6. Consider the following equation

$T_{\frac{2}{3}} y=\frac{\sqrt[3]{x}}{e^{x}} y^{2}+2 \sqrt[3]{x} y-2 \sqrt[3]{x} e^{x}$

A solution of this Riccati equation is $y_{1}=e^{x}$.

From (3.24), F and the change of variables from (3.25) are as the following form

$F(x)=e^{-4 x}, x=r, y=e^{r}-\frac{e^{4 r}}{s}$,

The original ODE becomes

$\frac{d s}{d r}=e^{3 r}$

The general solution of the Riccati fractional equation (3.29) is as follows

$y=e^{x}-\frac{e^{4 x}}{\frac{1}{3} e^{3 x}+C}$

\section{Conclusion}

In this paper, Lie Symmetry Analysis method have been applied for solving fractional differential equations, based on conformable fractional derivative. First order linear, Bernoulli and Riccati fractional differential equations, have been solved by the presented method. For each cases some examples are given for more explanation and clarification. The results showed that the presented method is easily applicable for this kind of equations.

\section{References}

[1] R. Khalil, M. A. Horani, A. Yousef and M. Sababheh, A new definition of fractional derivative, Journal of Computational and Applied Mathematics, $264 \quad$ (2014) https://doi.org/10.1016/j.cam.2014.01.002.

[2] T. Abdeljawad, on conformable fractional calculus, Journal of Computational and Applied Mathematics, 279 (2015) 57-66. https://doi.org/10.1016/j.cam.2014.10.016.

[3] Daniel J. Arrigo, Symmetry analysis of differential equations an introduction, 1nd Ed. John Wiley \& Sons, Inc. 2015.

[4] P. E. Hyden, Symmetry Methods for Differential Equations (A Beginner's Guide), Cambridge Texts in Applied Mathematics, 2000. https://doi.org/10.1017/CBO9780511623967.

[5] Peter J. Olver, Applications of Lie groups to differential equations, $2^{\text {nd }}$ Ed. Springer-Verlag, 1993.

[6] A. Ouhadan, E. H. EL. kinani, Exact solutions of time fractional Kolomogorov equation by using lie symmetry analysis, Journal of Fractional Calculus and Applications, 5 (1) (2014) 97-104.

[7] A. Elsaid, M. S. Abdel Latif, M. Maneea, Similarity solutions for solving Riesz fractional partial differential equations, Progress in Fractional Differentiation and Applications, 2 (4) (2016) 293-298. https://doi.org/10.18576/pfda/020407.

[8] Y. Zhanglie, Symmetry analysis to general time-fractional Korteweg-De Vries equations, Fractional Differential Calculus, 5 (2) (2015) 125-133. https://doi.org/10.7153/fdc-05-11.

[9] A. M. Yang, Y. Z. Zhang, C. Cattani, G. N. Xie, M. M. Rashidi, Y. J. Zhou, X. J. Yang, Application of local fractional series expansion method to solve Klein-Gordon equations on Cantor sets, Abstract and Applied Analysis, Volume 2014 (2014), Article ID 372741, 6 pages.

[10] S. Kumar, D. Kumar, S. Abbasbandy, M. M. Rashidi, Analytical solution of fractional Navier-Stokes equation by using modified Laplace decomposition method, Ain Shams Engineering Journal, 5 (2) (2014) 569-574. https://doi.org/10.1016/j.asej.2013.11.004.

[11] H. Khalil, R. Khan, M. M. Rashidi, Brenstien polynomials and its application to fractional differential equation, Computational methods for differential equations, 3 (1) (2015) 14-35.

[12] J. Singh, M. M. Rashidi, D. Kumar, R. Swroop, A fractional model of a dynamical Brusselator reaction-diffusion system arising in triple collision and enzymatic reactions, Nonlinear Engineering 5 (4) (2016) 277-285. https://doi.org/10.1515/nleng-2016-0041. 
[13] M. Gaur, K. Singh, Symmetry analysis of time-fractional potential Burgers' equation, Mathematical Communications, 22 (2017) 1-11.

[14] M. Gaur, K. Singh, Symmetry Classification and Exact Solutions of a Variable Coefficient Space-Time Fractional Potential Burgers' Equation, International Journal of Differential Equations, Article ID 4270724, 8 pages, (2016).

[15] M. Ilie, J. Biazar, Z. Ayati, General solution of Bernoulli and Riccati fractional differential equations based on conformable fractional derivative, International Journal of Applied Mathematical Research, 6(2) (2017) 49-51. https://doi.org/10.14419/ijamr.v6i2.7014.

[16] M. Ilie, J. Biazar, Z. Ayati, Application of the Lie Symmetry Analysis for second-order fractional differential equations, Iranian Journal of Optimization, 9(2) (2017) 79-83.

[17] M. Ilie, J. Biazar, Z. Ayati, Analytical solutions for conformable fractional Bratu-type equations, International Journal of Applied Mathematical Research, 7 (1) (2018) 15-19. https://doi.org/10.14419/ijamr.v7i1.8849.

[18] M. Ilie, J. Biazar, Z. Ayati, Optimal Homotopy Asymptotic Method for first-order conformable fractional differential equations, Journal of Fractional Calculus and Applications, 10 (1) (2019) 33-45.

[19] M. Ilie, J. Biazar, Z. Ayati, The first integral method for solving some conformable fractional differential equations, Optical and Quantum Electronics, 50 (2) (2018), https://doi.org/10.1007/s11082-017$\underline{1307-\mathrm{X}}$.

[20] M. Ilie, J. Biazar, Z. Ayati, Resonant solitons to the nonlinear Schrödinger equation with different forms of nonlinearities, Optik, 164 (2018) 201-209. https://doi.org/10.1016/j.ijleo.2018.03.013. 